\section{RISKS FACTORS FOR DEVELOPING NEONATAL PARENTERAL NUTRITION- ASSOCIATED CHOLESTASIS}

V. Champion ${ }^{1}$, I. Girard ${ }^{1}$, R. Carbajal ${ }^{2}$, J. Lozar ${ }^{3}$, S. Dahan ${ }^{4}$, E. Walter-Nicolet ${ }^{4}$, D. Mitanchez ${ }^{1}$

${ }^{1}$ Neonatology, ${ }^{2}$ Pediatric Emergency, Hôpital Armand Trousseau-Université Paris VI, Paris, France, ${ }^{3}$ University Children's Hospital, Ljubljana, Slovenia, ${ }^{4}$ Hôpital Armand Trousseau-Université Paris VI, Paris, France

Objective: To describe the incidence and the characteristics of cholestasis in a neonatal population dependent on parenteral nutrition (PN) and to analyse additional risk factors.

Methods: Monocentric prospective observational study conducted between April 2008 and April 2009. Bilirubinemia was weekly measured when PN was used. Cholestasis was defined as a conjugated hyperbilirubinemia $>17 \mu \mathrm{mol} / \mathrm{L}$ or $>20 \%$ of the total

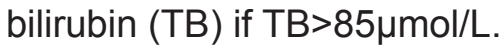

Results: 460 newborn were admitted in the unit. 207 required $\mathrm{PN}$. In this group, $28 \%$ were SGA, $33.8 \%$ were preterm $<32 \mathrm{WG}$ and $26.6 \%$ required surgery for abdomino-pelvic or thoracic pathologies. Cholestasis developed in 33 patients $(15.9 \%)$ at mean age of 14.2 days. No deaths were related. No cholestasis was noted in the others babies. In the NP group, the OR for developing cholestasis when born SGA or requiring surgery were 2.3 and 5.6. Prematurity $<32$ WG was not associated with cholestasis.

Perinatal asphyxia, pulmonary bronchodysplasia, secondary sepsis, length of $\mathrm{PN}$, protein, lipid, glucose daily intakes and the age at first enteral feeding were significantly related to cholestasis.

In multivariate analysis, cholestasis was associated to SGA ( $p=0.005)$, surgery $(p=0.003)$ and PN duration $(p<0.001)$. Protein daily intakes were not significantly associated with cholestasis.

Conclusion: Weekly bilirubinemia survey is indicated when $\mathrm{PN}$ is required $>7$ days, specially in SGA. Enteral nutrition should be started when enteral route is considered safe. There is no evidence to limit protein intakes.

\section{REGIONAL CEREBRAL OXYGEN SATURATION DURING NEONATAL TRANSITION - COMPARISON OF VAGINAL DELIVERY VS. CESARIAN SECTION}

\author{
B. Urlesberger, E. Kratky, M. Pocivalnik, \\ T. Rehak, W. Müller, G. Pichler
Div. Neonatology, Dept Pediatrics, Medical University Graz, Graz, Austria

Aim: The aim of the study was to investigate whether regional cerebral oxygen saturation (rSO2brain) shows significant differences in infants delivered by cesarian section (CS) compared to vaginal delivery (VD).

Methods: Prospective observational study. rSO2brain and preductal arterial oxygen saturation (SpO2) were measured during the first 15 minutes of life. Only healthy term infants with uncomplicated adaptation were included. Fractional tissue oxygen extraction (FTOE) was calculated.

Data are presented as mean. A linear mixed model with a fixed effect for time and a first order autoregressive covariance structure was used for calculation of significant differences.

Results: Between October 2009 and April 2010, 63 infants delivered by VD, and 29 infants delivered by elective CS were included into the study. SpO2 was $66 \%$ (CS)/73\% (VD) at minute 2, 81\%(CS)/89\%(VD) at minute $5,93 \%$ (CS)/95\%(VD) at minute 10 , and $95 \%(C S) / 97 \%(V D)$ at minute 15 . There was a significant difference in $\mathrm{SpO} 2$ data between VD and CS until minute 6 , showing higher values in VD group.

rSO2brain was $34 \%(\mathrm{CS}) / 39 \%(\mathrm{VD})$ at minute 2, $67 \%$ (CS)/69\%(VD) at minute $5,80 \%$ (CS)/77\%(VD) at minute 10 , and $80 \%(\mathrm{CS}) / 75 \%(\mathrm{VD})$ at minute 15. rSO2brain did not show any significant difference between $\mathrm{CS}$ and VD. FTOE was 0.49 (CS)/0.46(VD) at minute 2, 0.19(CS)/0.22(VD) at minute $5,0.13(\mathrm{CS}) / 0.19$ (VD) at minute 10 , and $0.16(\mathrm{CS}) / 0.22$ (VD) at minute 15 . FTOE did not show significant differences between CS and VD until minute 11, afterwards FTOE in VD group was higher.

Conclusion: rSO2brain was not constricted in CS group compared to VD group, which is in contrast to $\mathrm{SpO} 2$ behaviour. 